\title{
MAC Attack: Clinical Correlates of Mycobacterium avium Complex Infection Among Patients With and Without Cancer
}

\author{
Karan Gupta ${ }^{\mathrm{a}, \mathrm{c}}$, Marc B. Feinstein ${ }^{\mathrm{a}}$, Debra A. Goldman ${ }^{\mathrm{b}}$, \\ Hassan S. Patail ${ }^{\mathrm{a}}$, Diane E. Stover ${ }^{\mathrm{a}}$
}

\begin{abstract}
Background: In 2007 the American Thoracic Society (ATS) released guidelines on management of Mycobacterium avium complex (MAC), an increasingly common respiratory organism worldwide. Determining when this represents a true respiratory pathogen remains controversial and becomes increasingly challenging in patients with cancer. This study aims to 1) describe the phenotype that exists among cancer patients with MAC colonization and MAC pulmonary infection when compared to non-cancer patients; 2) assess whether cancer, symptoms, and radiographs, were associated with the decision to treat MAC pulmonary infection with antibiotics.
\end{abstract}

Methods: We retrospectively analyzed 550 adult, non-human immunodeficiency virus (HIV) patients, among whom MAC was identified in respiratory cultures or tissue. Radiographs, clinical symptoms and cancer status were studied. Patients were categorized as having MAC pulmonary infection based on 2007 ATS guidelines, and antibiotic treatment was thereafter reviewed. Fisher's exact test and Wilcoxon Rank sum assessed differences.

Results: Median age of the 550 patients was 68 years; most were female $(56 \%)$ and white $(83 \%)$. Symptoms and radiographic abnormalities accompanying MAC isolation were common, occurring among $83 \%$ and $99.6 \%$ respectively of all patients. There were 444 patients with MAC who had current or inactive cancers, most commonly hematologic (30\%) and lung (25\%) malignancies, while 106 patients never had cancer. Cancer patients were younger $(\mathrm{P}=$ $0.028)$, less often female $(\mathrm{P}<0.001)$, and had less-frequent pre-existing lung disease $(\mathrm{P}=0.017)$ than those without cancer. There were $196(35 \%)$ patients determined to have MAC pulmonary infection, among whom $49(9 \%)$ received directed antibiotics. Those receiving antibiotics had lower body mass index (BMI) $(\mathrm{P}<0.0001)$, more

Manuscript submitted December 5, 2019, accepted January 13, 2020

aPulmonary Division, Department of Medicine, Memorial Sloan-Kettering Cancer Center, New York, NY, USA

${ }^{b}$ Department of Epidemiology and Biostatistics, Memorial Sloan-Kettering Cancer Center, New York, NY, USA

${ }^{\mathrm{c} C}$ Corresponding Author: Karan Gupta, 1838 Thunder Mountain Drive, Henderson, NV 89012, USA. Email: kgupta0524@gmail.com

doi: https://doi.org/10.14740/jocmr4058 frequent pre-existing lung disease $(\mathrm{P}=0.003)$ and lower cancer rates $(\mathrm{P}=0.008)$ than those not receiving antibiotics. Patients receiving antibiotics were more likely to have cavitary disease $(\mathrm{P}=0.001)$, cough/dyspnea $(\mathrm{P}=0.012)$, hemoptysis $(\mathrm{P}<0.001)$, and constitutional symptoms $(\mathrm{P}=0.001)$.

Conclusions: In concordance with ATS guidelines, hemoptysis, constitutional symptoms, cough/dyspnea and cavitary disease were associated with highest likelihood to treat with antibiotics. The phenotype in cancer patients was quite different than the classic Lady Windermere syndrome. MAC pulmonary infection was treated less often in cancer patients. This study extends beyond the ATS guidelines to examine the potential import of malignancy on the colonization and potential treatment of MAC.

Keywords: Anti-bacterial agents; Lung diseases; Mycobacterium avium; Neoplasms; Nontuberculous mycobacteria; Respiratory tract infections; Tomography; Treatment outcome

\section{Introduction}

Mycobacterium avium complex (MAC) is ubiquitous and has become a common finding worldwide in respiratory secretions and tissues [1]. It accounts for over half of nontuberculous mycobacteria (NTM) isolates in North America and nearly $80 \%$ in Japan, with a rising global prevalence [1-4]. Determining when this isolation represents a true pathogen remains controversial despite treatment guidelines released in 2007 by the American Thoracic Society (ATS) [5, 6]. Clinical, radiographic, and microbiologic criteria are equally important, and all must be met to make a diagnosis of MAC pulmonary infection (MAC-PI) by these standards. Practically, this can be quite difficult. For example, symptoms of MAC-PI are often non-specific and include malaise, cough, dyspnea, weakness, and occasional hemoptysis [7]. Many patients can have concomitant bronchiectasis which itself can cause many similar symptoms and radiographs [8-10]. Furthermore, the diagnosis of MAC-PI does not always necessitate treatment [5]. Antibiotic courses are long, side effects are numerous, and relapse or reinfection are common [2].

The diagnosis and treatment of MAC infections can be 
even more challenging in the cancer patient. Symptoms associated with cancer and its treatment may be similar to those from MAC-PI. Whether the current published guidelines by the ATS should apply to cancer patients is unclear [5]. The overall likelihood of detecting respiratory MAC isolates among these patients may be affected by a selection bias. Patients at a cancer center are more closely surveilled for staging, treatment, and detection of metastases than is the population at large. For instance, Tamura et al (2016) demonstrated that the isolation of MAC was not uncommon among lung cancer patients, with $2 \%(25 / 1,258$ patients $)$ having MAC isolated at screening bronchoscopy [11]. Only 10 of these patients were ultimately determined to have active MAC-PI. Some studies imply that the presence of malignancy may not influence the decision to treat MAC. Desai et al (2012) suggested that cancer patients with asymptomatic MAC-PI may be followed without treatment, even among those receiving chemotherapy [12]. Additionally, among those treated, they observed that side effects sufficient to discontinue therapy were common $(56 \%$ of patients).

Broadening the scope further, infections of all types are the leading cause of mortality and morbidity in patients with cancer, occurring in nearly $46 \%$ of all cancer patients and $85 \%$ of neutropenic patients [13]. This can be due to the suppression of the immune system by both the treatment agents and sometimes the malignancy itself. In addition, advanced disease stage, neutropenia (for more than 7 days), indwelling lines, nosocomial exposure and radiation all increase the risk of infections in the cancer patient [13]. It has been shown that bacterial infections predominate across all cancer patients, and in those with neutropenia, opportunistic infections broaden to include fungal infections as well. However, there is little information on the susceptibility of cancer patients to atypical mycobacteria. Data suggesting any difference in mycobacterial infections in cancer patients compared to non-cancer patients are lacking, hence the reason for this study.

This current study seeks: 1) to describe the phenotype that exists among cancer patients with MAC colonization and MAC-PI when compared to non-cancer patients; 2) to assess whether cancer status, symptoms, and radiographs, were associated with the decision to treat MAC-PI with antibiotics.

\section{Materials and Methods}

This study retrospectively analyzed 550 adults without HIV infection, among whom MAC was isolated by sputum, bronchoscopy, or surgical lung biopsy between January 2012 and December 2014 at a tertiary cancer institution. Demographics, cancer history, radiographs, culture results, and treatments were reviewed.

Patients were then categorized as having MAC-PI based on clinical, radiologic, and microbiological criteria based on the 2007 ATS guidelines [5]. Based on these guidelines, clinical criteria were satisfied by the presence of cough/dyspnea, constitutional symptoms (fatigue, fever, night sweats and weight loss), hemoptysis and the appropriate exclusion of oth- er diagnoses. Radiographic criteria were satisfied by nodular (focal parenchymal) or cavitary opacities on chest radiograph, or a computed tomography (CT) scan that show multifocal bronchiectasis (airways disease) with multiple small nodules (diffuse parenchymal). Microbiologic criteria were satisfied in accordance with the 2007 ATS guidelines by: 1) at least two separate positive cultures from expectorated sputum; 2) positive culture from bronchoalveolar wash or lavage; or 3) either positive culture on transbronchial or other lung biopsy, or biopsy with mycobacterial histopathologic features (granulomatous inflammation or acid-fast bacilli) and at least one positive culture from sputum or bronchial wash. The remaining patients who did not meet these criteria were considered to have MAC colonization (MAC-COL).

Patient characteristics including age, gender, race, smoking history, BMI, pre-existing lung disease, and cancer status (none, active, inactive) were studied. History of any hematologic stem cell transplant and cancer type were also described. Cancers were characterized as inactive when patients had been cancer-free and without treatment for 2 or more years. Unless specified, prior and active cancers were combined under the term "cancer" given the heterogeneity in defining complete remission between various malignancies.

\section{Statistical analyses}

Patient characteristics, symptoms, and radiologic findings were stratified by cancer status and by receipt of antibiotics. Univariable comparisons were performed with Fisher's exact test and Wilcoxon Rank Sum test, where appropriate, and visualized with bar charts.

We used multivariable logistic regression to assess the relationship between patient characteristics, radiologic findings, and symptoms by receipt of antibiotics. Variables significant on univariable analyses at $\mathrm{P}<0.05$ were considered for multivariable analyses. To avoid overfitting due to the low number of events, we also used backward model selection with exit criterion of 0.05 to arrive at our final model. Two-sided $\mathrm{P}$ values less than 0.05 were considered statistically significant. All analyses were performed with SAS 9.4 (The SAS Institute, Cary, NC).

Ethical standards were met and the study protocol was approved by the Institutional Review Board at Memorial SloanKettering Cancer Center.

\section{Results}

This study included 550 patients, among whom MAC was initially isolated from respiratory sources including sputum, bronchoscopy (lavage or washing) or tissue biopsy cultures. Of these, 196 (36\%) met criteria for MAC-PI, with the remaining 354 patients considered to have MAC-COL. The median age was 68 years (range: 17 - 93 years). Demographic information is shown in Table 1 . There were slightly more female $(307 / 550,56 \%)$ than male patients $(243 / 550,44 \%)$, and the majority were white $(455 / 550,83 \%)$. Pre-existing lung dis- 
Table 1. Patient Characteristics Stratified by Any Cancer

\begin{tabular}{|c|c|c|c|c|}
\hline & \multicolumn{3}{|c|}{ Any cancer } & \multirow{2}{*}{ P value } \\
\hline & Total & No & Yes & \\
\hline Patients number & $550(100)$ & $106(19.3)$ & $444(80.7)$ & \\
\hline \multicolumn{5}{|l|}{ Gender } \\
\hline Male & $243(44.2)$ & $28(26.4)$ & $215(48.4)$ & $<0.001$ \\
\hline White & $455(82.7)$ & $91(85.8)$ & $364(82)$ & 0.70 \\
\hline Black & $19(3.5)$ & $2(1.9)$ & $17(3.8)$ & \\
\hline Asian & $56(10.2)$ & $11(10.4)$ & $45(10.1)$ & \\
\hline Other & $5(0.9)$ & $0(0)$ & $5(1.1)$ & \\
\hline Former & $280(50.9)$ & $55(51.9)$ & $225(50.7)$ & \\
\hline Current & $31(5.6)$ & $4(3.8)$ & $27(6.1)$ & \\
\hline BMI, median (range) & $24.2(12.3-57.0)$ & $24.2(13.7-49.4)$ & $24.3(12.3-57.0)$ & 0.79 \\
\hline \multicolumn{5}{|l|}{ Pre-existing lung disease } \\
\hline Yes & $283(51.5)$ & $66(62.3)$ & $217(48.9)$ & 0.017 \\
\hline No & $267(48.5)$ & $40(37.7)$ & $227(51.1)$ & \\
\hline \multicolumn{5}{|l|}{ MAC pulmonary infection } \\
\hline Yes & $196(35.6)$ & $65(61.3)$ & $131(29.5)$ & $<0.001$ \\
\hline No & $354(64.4)$ & $41(38.7)$ & $313(70.5)$ & \\
\hline
\end{tabular}

ease was present among roughly half of the patients $(283 / 550$, $51 \%)$. Among all patients, $444(81 \%)$ had any history of cancer, $157(29 \%)$ having inactive cancer, and $287(52 \%)$ having at least one active cancer. There were $106(19 \%)$ patients without cancer.

Patients with any history of cancer (i.e. active or inactive cancers) are compared to those without cancer (Table 1). Patients with cancer were younger compared to patients without cancer (median: 68 vs. 72 years, $\mathrm{P}=0.028$ ), less commonly female $(229 / 444,52 \%$ vs. 78/106, 74\%, P < 0.001), and less likely to have pre-existing lung disease (217/444, 49\% vs. $66 / 106,62 \%, \mathrm{P}=0.017)$. A lower proportion of patients with cancer had MAC-PI $(131 / 444,29 \%)$ compared to patients without cancer $(65 / 106,61 \%, \mathrm{P}<0.001)$. No other patient characteristics were significantly associated with cancer status $(\mathrm{P}=0.70$ - 0.79).

On a secondary subgroup analysis, patients with active cancer were compared against inactive and no cancer groups. It was found that active cancer patients were younger (median: 66 years, range: 17 - 90) compared to those with either inactive (median: 71 years, range; 21 - 93) or no cancer (median: 72, range: 27 - 89), $\mathrm{P}<0.001$. Additionally, a lower proportion of active cancer patients were female $(127 / 287,44 \%)$, followed by inactive $(102 / 157,65 \%)$ and no cancer $(78 / 106,74 \%), \mathrm{P}$
$<0.001$. Relatively few of the active cancer patients had preexisting lung disease $(107 / 287,37 \%)$ compared to either inactive $(110 / 157,70 \%)$ or no cancer $(66 / 106,62 \%), \mathrm{P}<0.001$. $\mathrm{BMI}$, smoking history, and race were not significantly associated with cancer status $(\mathrm{P}=0.36-0.95)$.

\section{Cancer characteristics}

Hematologic cancers were the most common in our cohort $(132 / 444,30 \%)$, followed by lung $(111 / 444,25 \%)$, breast $(55 / 444,12 \%)$, gastrointestinal $(55 / 444,12 \%)$, and genitourinary $(25 / 444,6 \%)$ cancers. Of those with hematologic cancers, 49 patients $(40 \%$ of hematologic cancers and $11 \%$ of all cancers) had undergone one or more hematologic stem cell transplants. The remaining patients had a variety of solid tumors. These findings are displayed in Figure 1.

\section{Symptoms}

Among all 550 patients, 459 (83\%) had at least one symptom that could be potentially attributable to MAC-PI. The most common were cough/dyspnea (402/550, 73\%), constitutional 


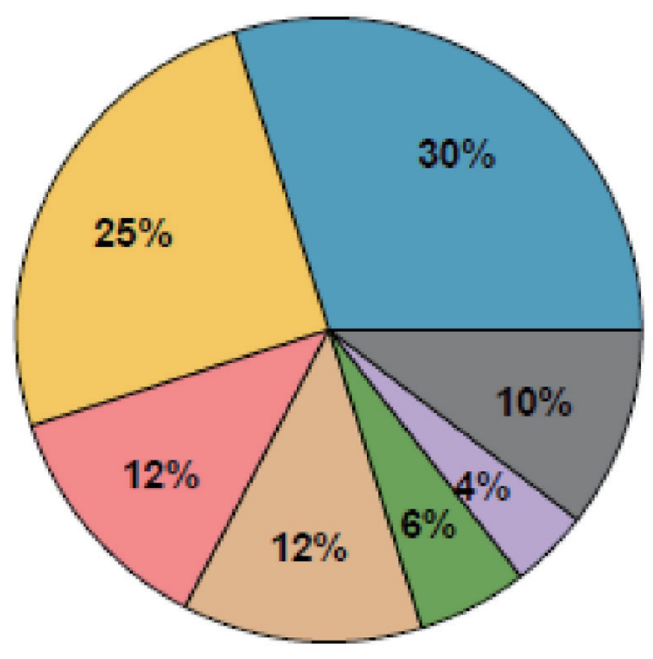

\section{$\square$ Hematologic $\square$ Lung $\square$ Breast $\square$ Gastrointestinal $\square$ Genitourinary $\square$ Head and Neck 口 Other}

Figure 1. Patient cancer characteristics.

symptoms $(96 / 550,17 \%)$ and hemoptysis $(51 / 550,9 \%)$. Respiratory failure was uncommon and present in 40 patients (7\%). Neutropenia was present among 46 patients $(8 \%)$ and leukocytosis among 56 patients $(10 \%)$.

\section{Radiologic findings}

All but two patients had at least one radiographic abnormality $(548 / 550,99.6 \%)$. The most common findings were focal parenchymal $(409 / 550,74 \%)$, diffuse parenchymal $(282 / 550$, $51 \%$ ), and airway disease $(147 / 550,27 \%)$. This is demonstrated with further description of each subset radiographic abnormality in Table 2.

\section{Associations of antibiotic treatment}

Forty-nine ( $9 \%$ of all patients and $25 \%$ of patients with MACPI) received antibiotics as treatment for MAC-PI. Comparisons to patients who did not receive antibiotics are shown in Table 3. Patients who received antibiotics had a lower BMI (21.5 vs. $24.7, \mathrm{P}<0.001$ ), higher rate of pre-existing lung disease $(71 \%$ vs. $50 \%, \mathrm{P}=0.004)$, and a lower rate of cancer $(67 \%$ vs. $82 \%, \mathrm{P}=0.021)$ compared to those who did not receive antibiotics. Patients receiving antibiotics more commonly experienced constitutional symptoms, cough/dyspnea, and hemoptysis as shown in Figure 2. No significant difference was seen in age, gender, race, or any history of smoking $(\mathrm{P}=0.18-0.47)$. No significant differences were noted in the rates of neutropenia, leukocytosis, respiratory failure, or chest
Table 2. Overall Radiologic Findings

\begin{tabular}{ll}
\hline Any radiology finding & $548(99.6)$ \\
Diffuse parenchymal & $282(51.3)$ \\
Focal parenchymal & $409(74.4)$ \\
Cavitary & $32(5.8)$ \\
Pleural disease & $49(8.9)$ \\
Airway disease & $147(26.7)$ \\
Lymphadenopathy & $85(15.5)$ \\
\hline
\end{tabular}

Diffuse parenchymal: ground glass opacities, airspace opacities, alveolar infiltrates, interstitial infiltrates, tree and bud infiltrates, mosaic pattern, mucus plugging, pulmonary edema, consolidation, emphysema, fibrosis, septal thickening. Focal parenchymal: nodule, mass, spiculated mass, granuloma, lung abscess. Pleural disease: pleural effusion, pleural thickening, hydropneumothorax, pneumothorax. Airway disease: atelectasis, bronchiectasis, endobronchial lesion, tracheomalacia, tracheobronchomalacia. Lymphadenopathy: mediastinal and hilar adenopathy.

pain $(\mathrm{P}=0.11-0.95)$.

Regarding radiographic findings, patients receiving antibiotics more commonly had cavitary lesions (17\% vs. $5 \%, \mathrm{P}$ $<0.001)$ and airways disease (predominantly bronchiectasis) (55\% vs. $24 \%, \mathrm{P}<0.001)$ on their imaging studies, and less commonly had lymphadenopathy ( $4 \%$ vs. $17 \%, \mathrm{P}=0.021)$ as shown in Figure 3. No significant differences were found in diffuse parenchymal, focal parenchymal or pleural disease $(\mathrm{P}$ $=0.29-0.30)$.

After backward selection in a multivariable model, cavitary disease (odds ratio (OR): 7.09, 95\% confidence interval (95\% CI): $2.70-18.60, \mathrm{P}<0.001)$, airway disease (OR: 4.62, 95\% CI: 2.33 - 9.16, P < 0.001), constitutional symptoms (OR: 3.80, 95\% CI: 1.87 - 7.73, P < 0.001), hemoptysis (OR: 3.34, $95 \% \mathrm{CI}: 1.51-7.39, \mathrm{P}=0.003)$ and cough/dyspnea (OR: 3.01, $95 \% \mathrm{CI}: 1.10-8.28, \mathrm{P}=0.032$ ) remained significant predictors of antibiotic treatment where presence of these symptoms or radiologic findings were associated with higher odds of receiving antibiotic treatment.

\section{Discussion}

The classic presentation of MAC-PI is the thin, elderly, nonsmoking woman with middle lobe and lingular atelectasis, sometimes referred to as Lady Windermere syndrome [4, 14, 15]. Patients described in this study with malignancy show a different phenotype than this classic description. Cancer patients who had MAC isolated (both MAC-PI and MAC-COL) were significantly younger than their counterparts without cancer, less often female, and less frequently had pre-existing lung disease. Typically considered a condition prevalent among woman, we found MAC-PI present at roughly equal rates among males and females with cancer. These key differences remained when cancer patients were divided into active vs. inactive, suggesting that patients in remission may behave more similarly to patients without cancer.

It is conceivable that, among at least some patients, cancer 
Table 3. Association Between Patient Characteristics and Antibiotics Treatment

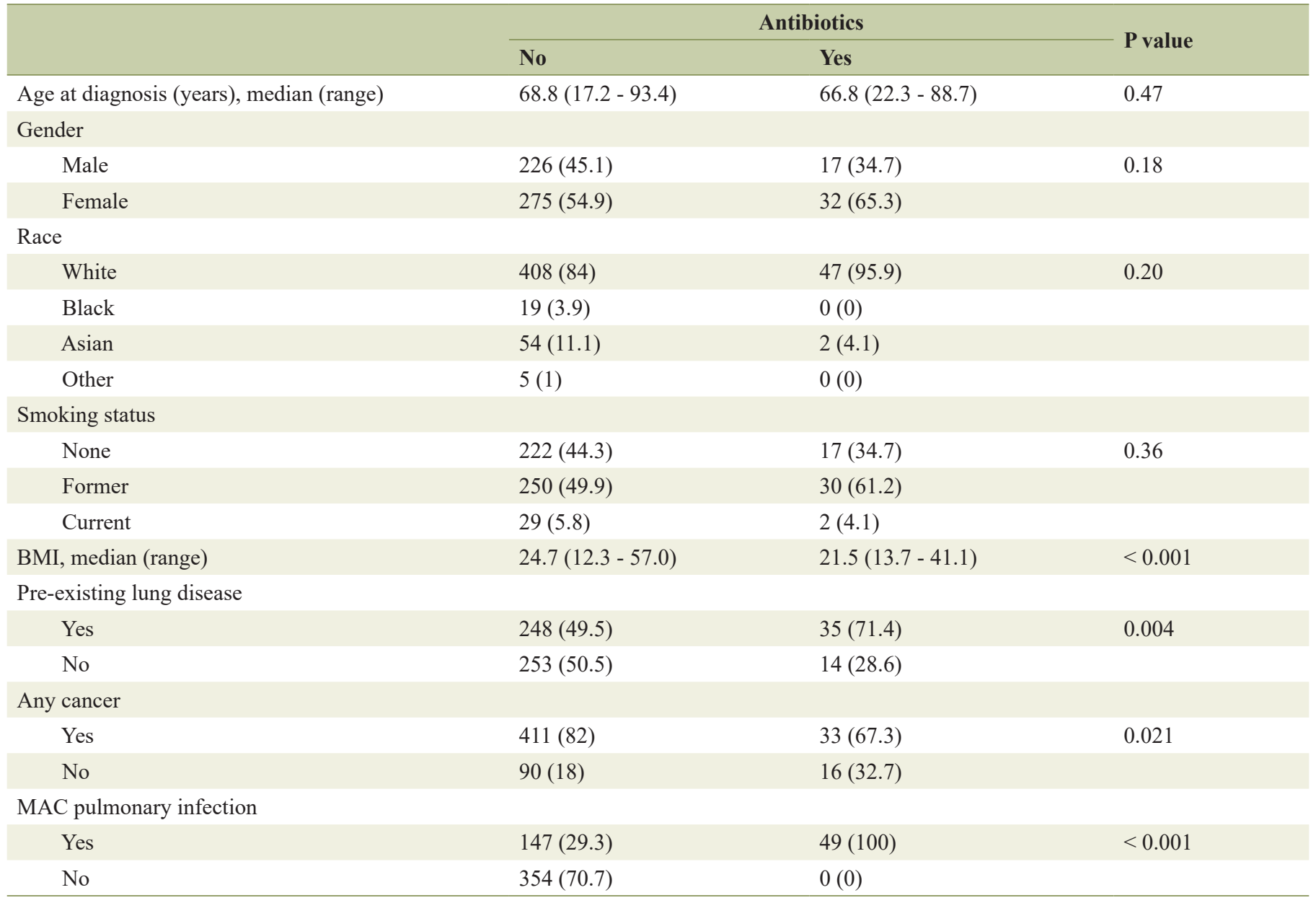

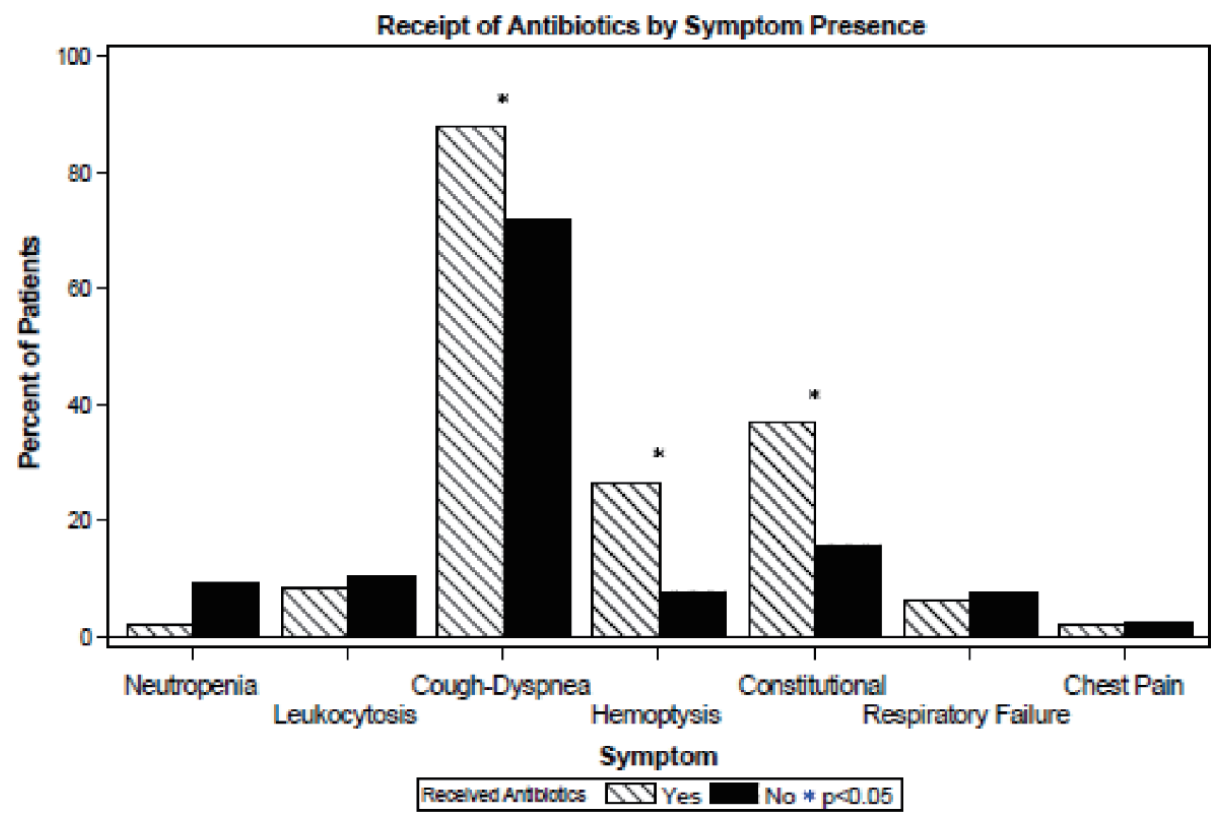

Figure 2. Symptom and lab characteristics of patients by receipt of antibiotics. 


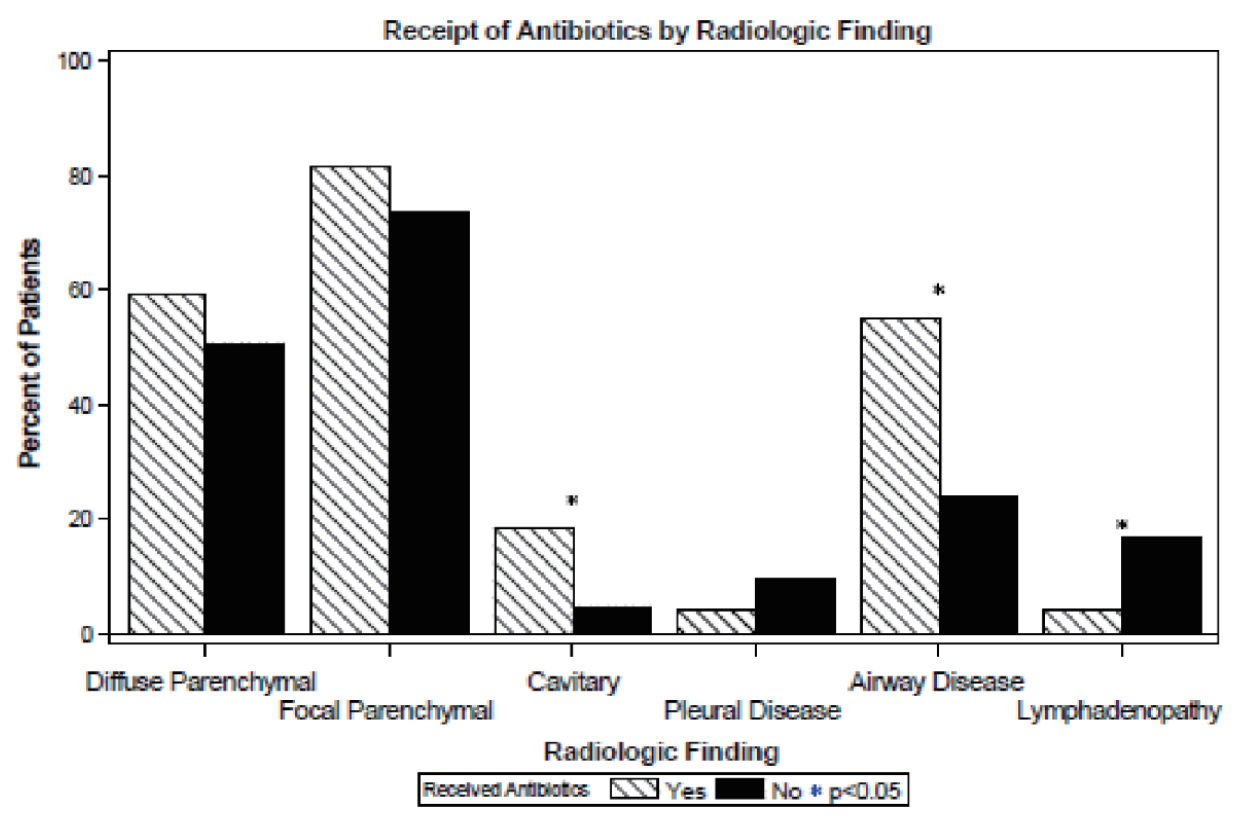

Figure 3. Radiologic findings of patients by receipt of antibiotics.

itself may be a risk factor for both colonization and infection with MAC; particularly for patients afflicted with a primary or metastatic tumor within the lungs. That architectural distortion from cancer or its treatment might predispose to MAC-PI is supported by the findings of Meier et al (2017), who demonstrated that among patients with lung cancer and MAC-PI, structural lung disease was present among eight of 13 patients [7]. Notably, four patients had no other apparent risk factors beyond their malignancy, which also propagates the concept of cancer as an independent risk factor for MACPI [7]. In addition, cachexia from cancer is associated with a continuous loss of locomotor skeletal muscle mass. This may cause profound muscle weakness, conceivably impairing mucociliary clearance, which in turn may contribute to both colonization and infection with organisms such as MAC [16].

Some studies have attempted to examine the import of MAC in patients with malignancy. For instance, Lai et al (2012) studied 219 cancer patients with various MAC infections and similarly found that the lungs were the most common site of infection [17]. Among these patients, and parallel to our findings, males were more often affected by MACCOL and MAC-PI in those with cancer. Additionally, they found lung and hematologic malignancies were most common amongst those with MAC-PI, consistent with our study. Weinstock et al (2003) found that 9.7\% of T-cell depleted and $7.0 \%$ conventional allogeneic stem cell transplant recipients had a positive culture for NTM after transplantation, and that rates of NTM infections were 5 to 20 -fold higher than previously reported at other centers [1]. Another group demonstrated that lung cancer was a comorbidity in $4-7 \%$ of patients with respiratory NTM (primarily MAC) isolates [11]. Unfortunately, it is beyond the scope of this study to determine and quantify to what extent cancer itself may be a risk factor for MAC.

Our study found that patients who received antibiotics for MAC-PI more frequently had hemoptysis, constitutional symptoms, cough/dyspnea and cavitary disease, congruent with current observations and ATS guidelines [5, 9]. Given the degree of immunosuppression from malignancy and treatment, one might suspect that cancer patients may be treated more frequently for MAC-PI. However, in our findings cancer patients with MAC-PI were treated in only $25 \%$ of cases, lower when compared to more generalized populations which approach nearly $60 \%$ treatment rates [18]. When treatments were pursued, most patients in our study were on combination antibiotics consistent with 2007 ATS guidelines, including macrolides (namely clarithromycin and azithromycin), ethambutol, rifampin and rifabutin. Intravenous and inhaled therapies were infrequent. Due to the retrospective nature of the study we were unable to standardize for regimen choices and duration of antibiotic therapy.

Several reasons may account for the interesting observation that MAC-PI was treated less often among patients that concurrently had cancer. First, due to the seriousness of their illness, cancer patients may receive more intensive medical care than those without cancer. The consequent selection bias might result in more respiratory cultures performed among relatively asymptomatic patients. Additionally, non-cancer patients with nodules and minimal symptoms may have been subjected to biopsies leading to diagnosis of MAC-PI more frequently than may have otherwise occurred because of evaluation at a cancer center. Second, impaired host defenses, secondary to malignancy or cancer therapy, may predispose to both colonization and infection. Third, symptoms and radiograph findings, which might ordinarily be attributed to MAC, may instead be attributed to other possibilities, including the malignancy itself, and/or toxicity from drugs or radiation, or alternative infections [3]. Finally, it is conceivable that clinicians simply have a higher threshold to submit cancer patients to the rigor of MAC treatment. Treatment of 
NTM may require a regimen of multiple drugs for 1 year or more, and side effects as well as drug interactions are common [5, 19]. In addition, re-treatment for relapse and/ or reinfection is required among approximately one-third of patients [2, 18]. Eradication rates have not been rigorously examined in cancer patients, but may vary from $55 \%$ up to $84 \%[6,18]$.

To our knowledge, our study has resulted in the one of the largest reviews that directly compares phenotypes and treatment rates of MAC in cancer vs. non-cancer counterparts at a single center. However, it is important to recognize some limitations of this study. It is retrospective in nature, and susceptible to limitations characteristic of this design. Hence, our results are reflective of who was treated with antibiotics in our cohort, as opposed to who should be treated. Patients were studied at a single urban, tertiary cancer center which may limit the ability to extrapolate results to other demographics. MAC cultures, detected at a cancer center, may have been found during suspected malignancy workups, implying that some evaluation might have been more aggressive than otherwise indicated for MAC alone. Dates of imaging studies could not be standardized in relation to dates of cultures. Initiation, duration and chosen regimen of antibiotics against MAC could also not be standardized.

Results described herein support the hypothesis that the decision to treat MAC-PI is driven primarily by symptoms and radiographs, in concordance with the 2007 ATS guidelines. However, we extend beyond these guidelines to examine the potential import of malignancy, deducing that the same criteria in diagnosis and treatment can and should be applied to the cancer population. Additionally, it appears clinicians should recognize that the phenotype in cancer patients is quite different than the classic Lady Windermere syndrome. Cancer patients with the isolation of MAC (both infection and colonization) were younger, less often female and less often had pre-existing lung disease. Interestingly, cancer patients with MAC-PI were overall treated less often than expected and when compared to generalized populations which can be accountable for a variety of reasons. Clinically, this becomes important as our findings may suggest that incidental MAC-positive cultures in the cancer patient may not be an immediate cause for concern and treatment. Instead, the overall decision to treat with antibiotics for MAC-PI was more likely, irrespective of cancer status, if hemoptysis, constitutional symptoms, cough/dyspnea and cavitary disease were present. Hence, further studies are warranted to determine to what extent cancer itself, as well as its associated treatments, are risk factors for the colonization and infection with MAC.

\section{Acknowledgments}

None to declare.

\section{Financial Disclosure}

None to declare.

\section{Conflict of Interest}

\author{
None to declare.
}

\section{Informed Consent}

This study was retrospective in its entirety and many of the patients had expired before data were collected. The study posed no physical harm to patients studied. Accordingly, the need for informed consent was waived by the Institutional Review Board at Memorial Sloan-Kettering Cancer Center. This study only used existing data, documents or specimens. No information pertaining to the identification of individual subjects was disclosed.

\section{Author Contributions}

Karan Gupta: design of work, acquisition of data, analysis and interpretation, composition, drafting, revising, editing, and final approval. Marc B. Feinstein: design of work, interpretation of data, drafting, revising, editing. Debra A. Goldman: analysis and interpretation of data, editing. Hassan S. Patail: acquisition of data, drafting, editing. Diane E. Stover: design of work, drafting, revising, editing, and final approval.

\section{Data Availability}

The authors declare that data supporting the findings of this study are available within the article.

\section{References}

1. Weinstock DM, Feinstein MB, Sepkowitz KA, Jakubowski A. High rates of infection and colonization by nontuberculous mycobacteria after allogeneic hematopoietic stem cell transplantation. Bone Marrow Transplant. 2003;31(11):1015-1021.

2. Jarand J, Davis JP, Cowie RL, Field SK, Fisher DA. Long-term follow-up of mycobacterium avium complex lung disease in patients treated with regimens including clofazimine and/or rifampin. Chest. 2016;149(5):12851293.

3. Prince DS, Peterson DD, Steiner RM, Gottlieb JE, Scott R, Israel HL, Figueroa WG, et al. Infection with Mycobacterium avium complex in patients without predisposing conditions. N Engl J Med. 1989;321(13):863-868.

4. Winthrop KL, McNelley E, Kendall B, Marshall-Olson A, Morris C, Cassidy M, Saulson A, et al. Pulmonary nontuberculous mycobacterial disease prevalence and clinical features: an emerging public health disease. Am J Respir Crit Care Med. 2010;182(7):977-982.

5. Griffith DE, Aksamit T, Brown-Elliott BA, Catanzaro A, Daley C, Gordin F, Holland SM, et al. An official ATS/ 
IDSA statement: diagnosis, treatment, and prevention of nontuberculous mycobacterial diseases. Am J Respir Crit Care Med. 2007;175(4):367-416.

6. Marras TK, Prevots DR, Jamieson FB, Winthrop KL, Pulmonary MACOG. Variable agreement among experts regarding Mycobacterium avium complex lung disease. Respirology. 2015;20(2):348-351.

7. Meier E, Pennington K, Gallo de Moraes A, Escalante P. Characteristics of Mycobacterium avium complex (MAC) pulmonary disease in previously treated lung cancer patients. Respir Med Case Rep. 2017;22:70-73.

8. Hayashi M, Takayanagi N, Kanauchi T, Miyahara Y, Yanagisawa T, Sugita Y. Prognostic factors of 634 HIV-negative patients with Mycobacterium avium complex lung disease. Am J Respir Crit Care Med. 2012;185(5):575-583.

9. Lee G, Lee KS, Moon JW, Koh WJ, Jeong BH, Jeong YJ, Kim HJ, et al. Nodular bronchiectatic Mycobacterium avium complex pulmonary disease. Natural course on serial computed tomographic scans. Ann Am Thorac Soc. 2013;10(4):299-306.

10. Wittram C, Weisbrod GL. Mycobacterium avium complex lung disease in immunocompetent patients: radiography-CT correlation. Br J Radiol. 2002;75(892):340344.

11. Tamura A, Hebisawa A, Kusaka K, Hirose T, Suzuki J, Yamane A, Nagai H, et al. Relationship Between Lung Cancer and Mycobacterium Avium Complex Isolated Using Bronchoscopy. Open Respir Med J. 2016;10:20-28.

12. Desai D, Adachi JA, Rolston K. Asymptomatic Non-tuberculous Mycobacterial (NTM) lung infections in cancer patients: MD Anderson Experience. Houston, Texas. 2012. Poster abstract.
13. Calik Basaran N, Karaagaoglu E, Hascelik G, Durusu Tanriover M, Akova M. Prospective evaluation of infection episodes in cancer patients in a tertiary care academic center: microbiological features and risk factors for mortality. Turk J Haematol. 2016;33(4):311-319.

14. Reich JM, Johnson RE. Mycobacterium avium complex pulmonary disease presenting as an isolated lingular or middle lobe pattern. The Lady Windermere syndrome. Chest. 1992;101(6):1605-1609.

15. Yamazaki Y, Kubo K, Takamizawa A, Yamamoto H, Honda T, Sone S. Markers indicating deterioration of pulmonary Mycobacterium avium-intracellulare infection. Am J Respir Crit Care Med. 1999;160(6):1851-1855.

16. Roberts BM, Ahn B, Smuder AJ, Al-Rajhi M, Gill LC, Beharry AW, Powers SK, et al. Diaphragm and ventilatory dysfunction during cancer cachexia. FASEB J. 2013;27(7):2600-2610.

17. Lai CC, Tan CK, Cheng A, Chung KP, Chen CY, Liao $\mathrm{CH}$, Huang YT, et al. Nontuberculous mycobacterial infections in cancer patients in a medical center in Taiwan, 2005-2008. Diagn Microbiol Infect Dis. 2012;72(2):161165.

18. Lee BY, Kim S, Hong Y, Lee SD, Kim WS, Kim DS, Shim TS, et al. Risk factors for recurrence after successful treatment of Mycobacterium avium complex lung disease. Antimicrob Agents Chemother. 2015;59(6):29722977.

19. Wallace RJ, Jr., Brown-Elliott BA, McNulty S, Philley JV, Killingley J, Wilson RW, York DS, et al. Macrolide/ Azalide therapy for nodular/bronchiectatic mycobacterium avium complex lung disease. Chest. 2014;146(2):276282. 